\title{
Influence of Patients' Adherence to Medication, Patient Background and Physicians' Compliance to the Guidelines on Asthma Control
}

\author{
Tomoko SUZUKI, ${ }^{*, a, b}$ Isao SAITO, ${ }^{a}$ Mitsuru ADACHI,${ }^{c}$ Takuro Shimbo, ${ }^{b}$ and Hitoshi SATO ${ }^{a}$ \\ aDeparment of Pharmacokinetics and Pharmacodynamics, Faculty of Pharmaceutical Sciences, Showa \\ University, 1-5-8 Hatanodai, Shinagawa-ku, Tokyo 142-8555, Japan, bepartment of Clinical \\ Research and Informatics, Research Institute National Center for Global Health and Medicine, \\ 1-21-3 Toyama, Shinjuku-ku, Tokyo 162-8655, Japan, and ${ }^{c}$ First Department of Internal \\ Medicine, Showa University, 1-5-8 Hatanodai, Shinagawa-ku, Tokyo 142-8555, Japan
}

(Received May 23, 2010; Accepted October 3, 2010; Published online October 8, 2010)

\begin{abstract}
This study conducted a thorough examination on the associations among several factors of asthma therapy, i.e., physicians' compliance with the guidelines, patients' adherence to medication, asthma symptom severity, asthma episodes, and patient background. Fifty outpatients treated continuously for asthma from October 2002 to October 2004 at Showa University Hospital were selected and their medical charts were surveyed. Physicians were recognized as noncompliant when their treatments included divergence from the Asthma Prevention and Management Guidelines. Patient adherence was evaluated as the ratio of the "measured (dispensed)" doses divided by the "expected (prescribed)" doses. The inhaled corticosteroids adherence and the ratio of the asthma-related emergency department visits of the patients with a family asthma medical history were significantly higher $(p=0.034)$ and lower $(p=0.043)$, respectively, than those without this medical history. This may indicate the necessity of education for patients with no family history of asthma. A significant correlation between the mean patients' adherence and the asthma symptom severity at the end of surveillance was found $(p=0.010)$, suggesting the importance of patient adherence in asthma control. The asthma symptom severity in the noncompliant group at the beginning of surveillance was significantly worse $(p=0.016)$, suggesting that physician compliance was low when the asthma symptom severity was poor. Based on the above findings, we proposed a flow-chart, which includes the confirmation processes of patients' adherence to medication and physicians' compliance to guidelines, in order to better control asthma, while also taking their family medical history into account.
\end{abstract}

Key words_— asthma control; family history; patients' adherence; physicians' compliance; symptom severity; background

\section{INTRODUCTION}

Clinical guidelines help practitioners diagnose or make decisions regarding the appropriate health care under specific circumstances. The Global Initiative for Asthma (GINA) was updated in 2008.1) The Japanese guidelines for the management of asthma, the Asthma Prevention and Management Guidelines in Japan (JGL1998) have been updated as JGL2000, JGL2003 and JGL2006. ${ }^{2)}$

Domestic and international guidelines recommend the use of inhaled steroids for the treatment of asthma as a first-line therapy, particularly in the treatment of mild-to-severe persistent asthma in adults. Inhaled corticosteroids (ICS) are found to be effective in controlling the symptoms of asthma and reducing asthma mortality rates..$^{3)}$ Many studies have demonstrated the benefits of inhaled steroids to control asthmatic

\footnotetext{
*e-mail: tsuzuki-nii@umin.net
}

symptoms. de Marco et al. ${ }^{4)}$ reported that there was a positive correlation between a good control of the disease and an adequate dose of ICS, which followed the GINA recommendations. They also reported that the patients treated without conformity to the guidelines experienced significantly more asthmatic attacks, thus indicating the importance of the compliance with the guidelines.

Despite the above-mentioned advantages of asthma guidelines, patient medication nonadherence will lead to a failure of asthma control. Williams et al..$^{5)}$ found that adherence to ICS was negatively correlated with the number of emergency department visits. Poor adherence to ICS therefore appears to account for the majority of asthma-related hospitalizations. We have previously reported that fluticasone propionate (FP) inhalation was more effective than a two-fold higher dosage of beclomethasone dipropionate inhalation, and that a better FP adherence contributed to its efficacy, most likely due to improved factors associat- 
ed with FP adherence. ${ }^{6}$

Patient nonadherence with medication regimens and physicians' noncompliance with the guidelines are not a disease-specific but a general problem, and are crucial factors for the treatment of diseases. Therefore, it is important to monitor and improve the status of medication adherence and physician guideline compliance.

Patient background data contain important factors related to asthma therapy. Barr et al. ${ }^{7)}$ reported that lower socioeconomic status, severity of asthma, current smoking, and an earlier onset of asthma were associated with patient nonadherence with medication regimens. We have also reported that an earlier onset of asthma and a longer asthma duration were associated with nonadherence. ${ }^{8)}$

So far, however, there have been no reports that thoroughly investigate the associations among these important factors of asthma therapy, i.e., asthma control, physician guideline compliance, and patient medication adherence and background, although some of these correlations have been suggested. The present study was designed to investigate the statistical associations among these factors of asthma therapy. Asthma control was evaluated by asthma symptom severity and asthma episodes (asthma-related emergency department visits and asthma-related unscheduled emergency visits). We also performed a causality analysis for the above-mentioned factors.

\section{METHODS}

Study Design All outpatients treated continuously for asthma (aged 16-50 years of age) at Showa University Hospital (Tokyo, Japan) were selected, and their medical charts were surveyed. The term of the surveillance was from October 2002 to October 2004. The outpatients who regularly visited the hospital for more than 1 year were selected as subjects in the present study. Because the asthma treatments for children under the age of 16 are different from those for adults, and prescriptions for patients aged 51 or over are often affected by medicines other than those administered for asthma, the subjects were selected within these age limits.

For measuring patient ICS adherence, we employed the ratio of "measured (dispensed)" doses divided by " "expected (prescribed)", doses.9,10) To calculate the "expected" doses, we used the prescribed physician orders which were documented in the medical charts. The "measured" doses were calculated from the doses which were actually written on the prescriptions by physicians and dispensed in the pharmacy. In the case that the "expected" doses were equal to the " "measured" doses, adherence becomes unity, which corresponds to a good adherence. Similarly, the adherence less than 1 suggests that the patient inhales the doses under the prescribed doses.

Asthma symptom severity and physician guideline compliance were checked by every patient visit based on the information described in medical charts, such as prescriptions, peak expiratory flows, and symptoms. As for the physicians' compliance or noncompliance, we assessed whether the treatment for all patient visits was in conformity to the JGL; physicians were recognized as noncompliant if their treatments included a divergence from the JGL. For example, a physician who prescribed only ICS for a patient with severe persistent asthma was judged to be noncompliant, since other drugs should be administered concomitantly with ICS based on the guidelines.

To investigate the statistical associations among some factors of asthma therapy control, the data at the beginning and the end of the surveillance were analyzed when they could feasibly be measured. The data at the beginning and at the end of the surveillance were the data collected at the time of the first and the last visits during the surveillance.

With respect to ethical considerations, the protection of privacy and personal information was ensured by using the anonymity linking capability of the patients. The institutional review board of Showa University approved the study protocol.

The following evaluations were analyzed for each patient: gender, previous history, family asthma medical history and smoking habits (Table 1).

1. Patients' ICS adherence was categorized by each characteristic of the patients (Fig. 1).

2. Each asthma episode was categorized by each characteristic of the patients (Fig. 2).

3. The distribution of asthma symptom severity was categorized by each characteristic of the patients (Table 2) .

4. Associations between patients' ICS adherence and asthma symptom severity at the (a) beginning or (b) end of surveillance were evaluated to identify the causes and consequences.

5. Asthma symptom severities at the beginning and the end of surveillance for the compliant and non- 
Table 1. Characteristics of the Patients Selected in the Present Study

\begin{tabular}{|c|c|c|c|c|}
\hline & \multirow{2}{*}{ Total } & \multicolumn{2}{|c|}{ Asthma medical history in their family } & \multirow{2}{*}{$p$} \\
\hline & & Without & With & \\
\hline No. of patients & 50 & $34(71 \%)$ & $14(29 \%)$ & \\
\hline Age in years & $36.3 \pm 7.9$ & $36.1 \pm 8.4$ & $36.9 \pm 7.5$ & 0.751 \\
\hline Gender ratio men/women (\%) & $27 / 23(54 \% / 46 \%)$ & $20 / 14(59 \% / 41 \%)$ & $6 / 8(43 \% / 57 \%)$ & 0.355 \\
\hline Body height $(\mathrm{cm})$ & $164.2 \pm 9.2$ & $164.1 \pm 9.8$ & $164.1 \pm 8.1$ & 0.937 \\
\hline Body weight (kg) & $59.4 \pm 11.3$ & $59.2 \pm 11.6$ & $59.8 \pm 11.5$ & 0.981 \\
\hline $\begin{array}{l}\text { No. of patients with concomitant disease } \\
(\%)\end{array}$ & $\begin{array}{l}2(4 \%) \\
\text { (hyperlipidemia 1, fatty } \\
\text { liver and hypertension 1) }\end{array}$ & $2(6 \%)$ & $0(0 \%)$ & 1.000 \\
\hline $\begin{array}{l}\text { No. of patients with previous history } \\
(\%)\end{array}$ & $34(68 \%)$ & $22(65 \%)$ & $11(79 \%)$ & 0.498 \\
\hline $\begin{array}{l}\text { No. of patients with aspirin-induced } \\
\text { asthma }(\%)\end{array}$ & $2(4 \%)$ & & & \\
\hline $\begin{array}{l}\text { No. of patients with atopic dermatitis } \\
(\%)\end{array}$ & $9(18 \%)$ & & & \\
\hline $\begin{array}{l}\text { No. of patients with allergic rhinitis } \\
(\%)\end{array}$ & $23(46 \%)$ & & & \\
\hline \multicolumn{5}{|l|}{ Smoking habits } \\
\hline $\begin{array}{l}\text { No. of patients } \\
\text { (non-smoker: past smokers : current } \\
\text { smokers) }(\%)\end{array}$ & $\begin{array}{c}31: 8: 10 \\
(63 \%: 16 \%: 21 \%)\end{array}$ & $\begin{array}{c}22: 7: 5 \\
(65 \%: 21 \%: 14 \%)\end{array}$ & $\begin{array}{c}9: 1: 4 \\
(64 \%: 7 \%: 29 \%)\end{array}$ & 1.000 \\
\hline $\begin{array}{l}\text { No. of patients on the day of the initial } \\
\text { visit with respect to asthma symptom } \\
\text { severity } \\
\text { (Mild intermittent : Mild persistent : } \\
\text { Moderate persistent : Severe persistent) } \\
\text { (\%) }\end{array}$ & $\begin{aligned} 3: 11 & : 24: 12 \\
(6 \%: 22 \% & : 48 \%: 24 \%)\end{aligned}$ & $\begin{array}{c}3: 5: 16: 7 \\
(10 \%: 16 \%: 52 \%: 22 \%)\end{array}$ & $\begin{array}{c}0: 6: 6: 2 \\
(0 \%: 43 \%: 43 \%: 14 \%)\end{array}$ & 0.383 \\
\hline
\end{tabular}

compliant groups were evaluated to know which is the cause and consequence.

The asthma symptom severity index was classified into mild intermittent, mild persistent, moderate persistent, and severe persistent in accordance with the severity classification in the JGL guidelines, and were analyzed as the ordinal variables 1, 2, 3, and 4, respectively. The Spearman rank correlation test, the Mann-Whitney U test, Fisher's exact test, and the Wilcoxon sign rank test were used for the statistical analyses using the JMP version 7 software program (SAS Institute, Cary, NC, USA). There were few missing values. An observation was omitted from the analysis if it has a missing value. Differences with $p$ values of less than 0.05 were considered to be statistically significant.

\section{RESULTS}

Fifty outpatients treated continuously for asthma from October 2002 to October 2004 at Showa University Hospital were selected, and the patient charac- teristics are shown in Table 1. The mean values of each patient characteristic between 2 groups, with and without asthma medical history in their family are also shown in Table 1. Each background factor of patient was independent of the family asthma medical history.

The observation period ranged from 17 to 27 months (mean time of 24 months). Forty two patients were prescribed with ICS continuously. The mean patients' adherence to medication and asthma symptom severity were $0.84 \pm 0.39(n=42$, mean \pm S.D.; median 0.74 , interquartile range $(25 \%-75 \%)$, $0.62-1.01)$ and $2.79 \pm 0.85(n=50$, mean \pm S.D.; median 2.77 , interquartile range, 2.12-3.59), respectively. The percentage of patients with guideline-compliant drug therapy was $78.0 \%$.

The influence of the background factors of patients on asthma control was investigated. The median values of ICS adherence in a patient without family asthma history and in a patient with a family asthma history were calculated to be 0.70 and 1.01 , respec- 

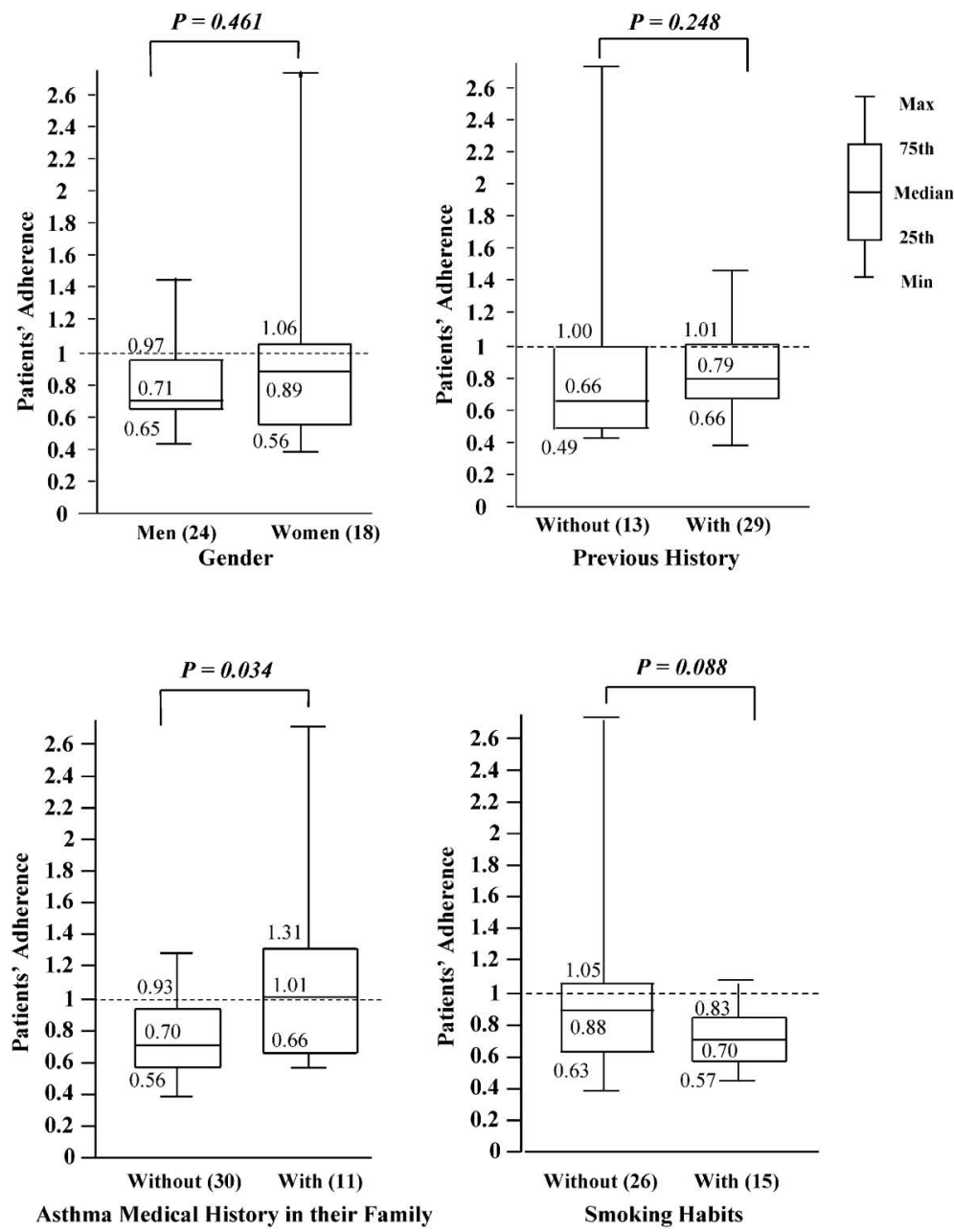

Fig. 1. Patients' ICS Adherence Categorized by Each Characteristic of the Patients

The Mann-Whitney U test was applied to compare the patients' ICS adherence between the 2 groups categorized by each characteristic of the patients. The boxplots indicate: the end of the upper tail, maximal value; end of the lower tail, minimum value; upper limit of the box, 75th percentile value; lower limit of the box, 25 th percentile value; horizontal line through box, median value. The number in the parentheses shows the number of cases that were analyzed. The horizontal dotted line shows 1.0 of the patients' adherence, which is the proper level of adherence.

tively. The ICS adherence of the patients with an asthma medical history in their family was significantly higher than those without such a medical history $(p$ $=0.034$, Mann-Whitney U test). Other characteristics (i.e. gender, previous history, smoking habits) were found not to be the significant influencing factors (Fig. 1).

The ratio of the asthma-related unscheduled emergency visits in patients with asthma medical history in their family tended to be lower than those without a family medical history of asthma $(14.3 \%$ and $32.4 \%$, respectively. $p=0.292$ by Fisher's exact test, Fig. 2 (a)). The ratio of the asthma-related emergency department visits in patients with asthma medical history in their family was significantly lower than for those patients who did not have a family medical history of asthma $(0.0 \%$ and $26.5 \%$, respectively. $p$
$=0.043$ by Fisher's exact test, Fig. 2(b)). Other characteristics (i.e., gender, previous history, and smoking habits) had no significant influence on the number of emergency department visits (Fig. 2).

The median asthma symptom severities of the patients with and without smoking habits were 3.00 (interquartile range, 2.69-4.00) and 2.15 (interquartile range, 2.00-3.25), respectively. The severity of asthma symptoms was significantly worse in the patients with smoking habits $(p=0.005$, Mann-Whitney $\mathrm{U}$ test). Other characteristics (i.e., gender, previous history, and asthma medical history in their family) were not found to be significant factors (Table 2).

In general, patient adherence was estimated to be good if the adherence ranged from 0.7 to $1.25 .^{8)}$ While the correlation between the mean patients' adherence to medication and the asthma symptom 
(a)

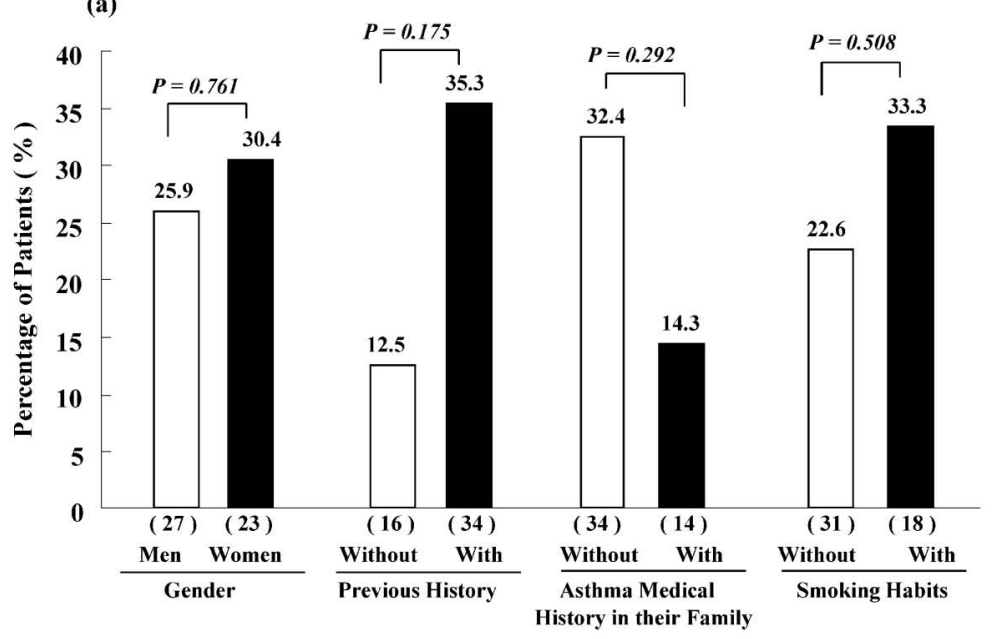

(b)

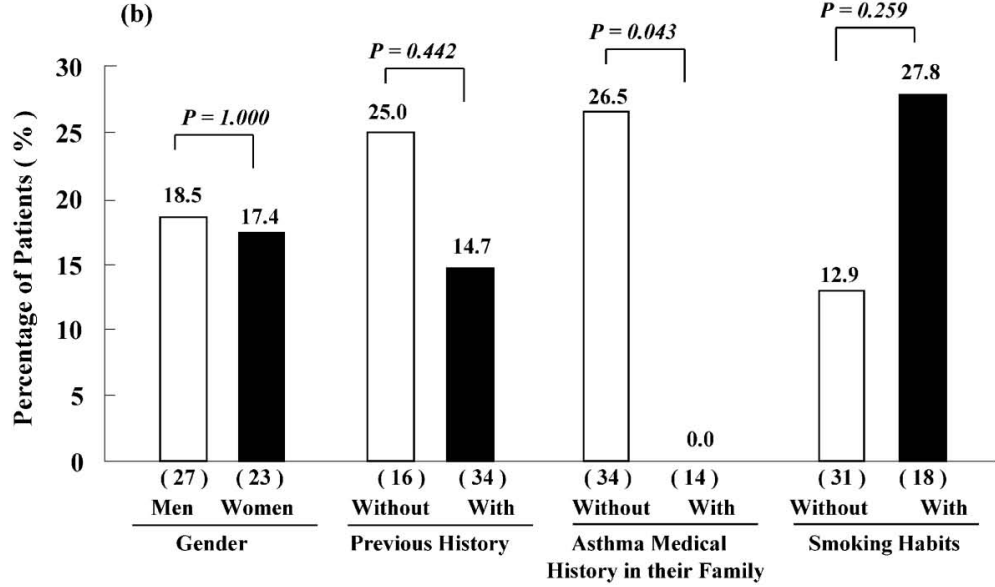

Fig. 2. Each Asthma Episode Categorized by Each Characteristic of the Patients

The comparison of the respective percentage of outpatients treated in (a) an asthma-related unscheduled emergency or (b) the emergency department. The number in the parentheses shows the number of patients in each 2 groups as categorized by each patient characteristic. The comparisons of the respective number of patients who were treated in an asthma-related unscheduled emergency or emergency department, regarding each of the 2 groups as categorized by each characteristic of the patients, were performed by Fisher's exact test.

Table 2. Distribution of Asthma Symptom Severity Categorized by Each Characteristic of the Patients

\begin{tabular}{|c|c|c|c|c|}
\hline Characteristics of the patients & & $n$ & $\begin{array}{l}\text { Asthma symptom severity } \\
\text { Median }(25 \%-75 \%)\end{array}$ & $p$ \\
\hline \multirow{2}{*}{ Gender } & men & 27 & $3.00(2.10-4.00)$ & \multirow{2}{*}{0.438} \\
\hline & women & 23 & $2.67(2.14-3.00)$ & \\
\hline \multirow{2}{*}{ Previous history } & without & 16 & $2.4(2.16-3.00)$ & \multirow{2}{*}{0.557} \\
\hline & with & 34 & $2.95(2.08-4.00)$ & \\
\hline \multirow{2}{*}{ Asthma medical history in their family } & without & 34 & $3.00(2.14-3.88)$ & \multirow{2}{*}{0.134} \\
\hline & with & 14 & $2.41(2.00-3.00)$ & \\
\hline \multirow{2}{*}{ Smoking habits } & without & 31 & $2.15(2.00-3.25)$ & \multirow{2}{*}{0.005} \\
\hline & with & 18 & $3.00(2.69-4.00)$ & \\
\hline
\end{tabular}

The results were presented as medians and interquartile ranges $(25 \%, 75 \%)$. Mann-Whitney U test was applied to compare asthma symptom severity between 2 groups. 
(a)

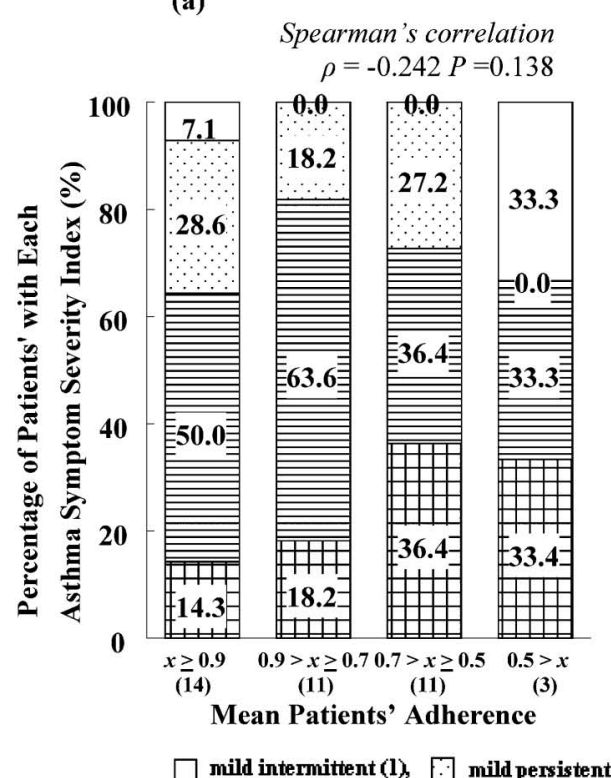

(b)

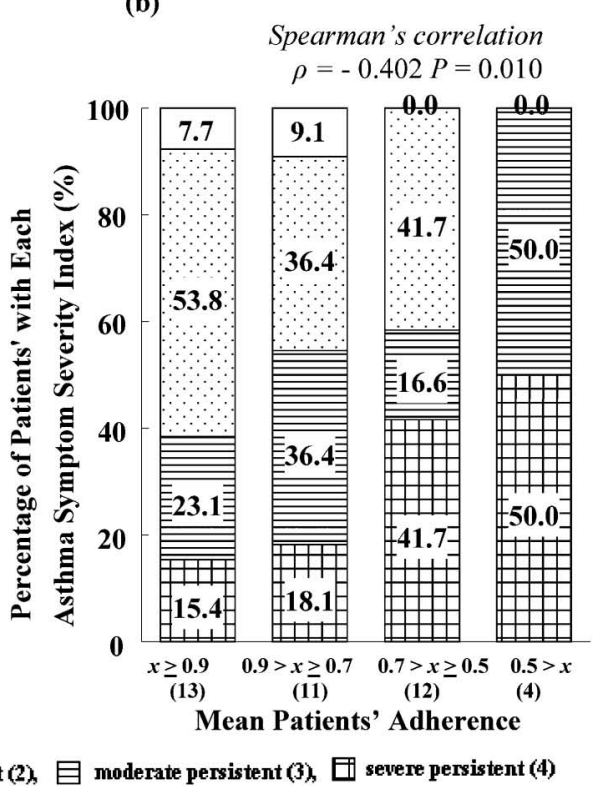

Fig. 3. Association between Mean Patients' Adherence to Medication and Asthma Symptom Severity at the Beginning (a) and End (b) of Surveillance

The patient asthma symptom severity index was classified into mild intermittent (1), mild persistent (2), moderate persistent (3) and severe persistent (4). Spearman's rank correlation test was employed to evaluate the association. The number in the parentheses shows the number of cases that were analyzed.

severity at the beginning of the surveillance period was not significant (the Spearman's correlation coefficients $\rho=-0.242, p=0.138$, Fig. 3 (a)), a significant correlation between the mean patients' adherence and the asthma symptom severity at the end was found out ( $\rho=-0.402, p=0.010$, Fig. $3(\mathrm{~b})$ ). The better the patients' adherence was, the more improved the asthma symptom severity. The mean difference of the asthma symptom severity between the end and the beginning of the surveillance was found to be $-0.26 \pm 0.17$ in the "good" adherence group with a greater than 0.7 adherence value. The difference was $0.071 \pm 0.25$ in the "poor" adherence group, with a less than 0.7 adherence value. The asthma symptom severity improved in the good adherence group, but it did not improve in the group with poor adherence, though this trend was not found to be statistically significant (Mann-Whitney U test).

The asthma symptom severity was also compared in the guideline-compliant and -noncompliant groups. The severities of asthma symptom at the beginning of the surveillance were $2.7 \pm 0.14$ (mean \pm S.E. interquartile range, 2-3) and $3.4 \pm 0.16$ (mean \pm S.E. interquartile range, 3-4) in the compliant and noncompliant groups, respectively. Of each asthma symptom severity index at the beginning of the surveillance, the ratios of moderate persistent and severe persistent were higher, and mild intermittent and mild persistent were lower in the noncompliant group than in the compliant group. The severity of symptoms in the noncompliant group at the beginning of the surveillance was significantly worse than that in the compliant group ( $p=0.016$, Mann-Whitney U test, Fig. 4). Conversely, the values at the end of the surveillance were $2.7 \pm 0.16$ (mean \pm S.E. interquartile range, 2-3.75) and $3.0 \pm 0.26$ (mean \pm S.E. interquartile range, 2-4) in the compliant and noncompliant groups, but this difference was not statistically significant ( $p=0.312$, Mann-Whitney U test). Furthermore, the asthma symptom severity in the noncompliant group showed no significant difference between the two periods of surveillance, i.e., the beginning and the end ( $p=0.250$, Wilcoxon sign rank test). These results suggest that physician compliance is low when the level of patient asthma symptoms severity is poor, although the asthma symptom severity is not poor when the physicians' compliance is low.

\section{DISCUSSION}

The present study conducted a thorough investigation of the associations among several factors of asthma therapy, i.e., physicians' compliance with the guidelines, patients' adherence to medication, asthma symptom severity, asthma episodes, and patient back- 


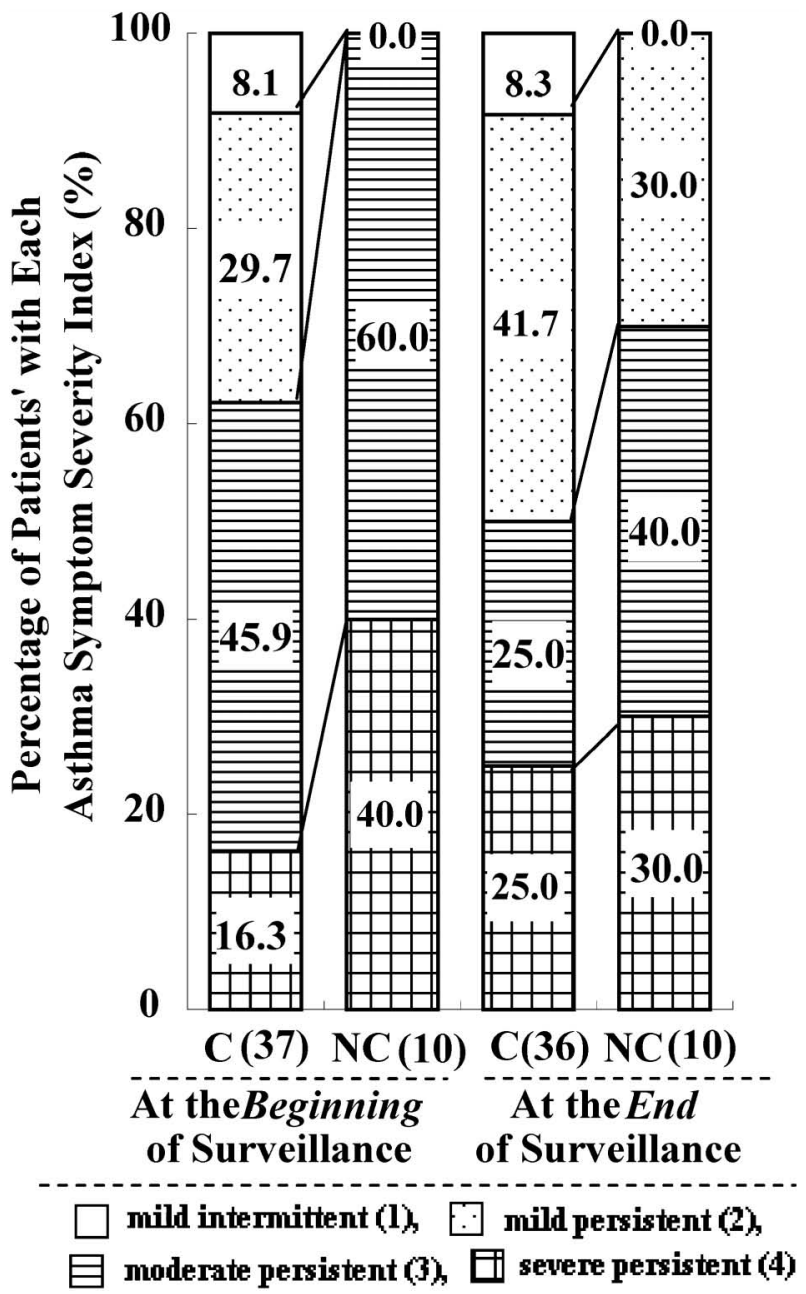

Fig. 4. Asthma Symptom Severities at the Beginning and the End of Surveillance for the Compliant and Noncompliant Groups

$\mathrm{C}$ and NC denote the compliant and noncompliant groups, respectively. Data were analyzed by using the Mann-Whitney U test. The asthma symptom severity index was classified into mild intermittent (1), mild persistent (2), moderate persistent (3) and severe persistent (4). The number in the parentheses shows the number of cases that were analyzed.

ground.

Lagerløv et al. ${ }^{11)}$ found that most physicians were apparently in agreement with guideline recommendations for asthma treatment. However, the proportion of asthma patients receiving inhaled steroids remained approximately $44 \%(31-58 \%),{ }^{11)}$ thus suggesting that physician recognition of the importance of inflammatory components of asthma is still not sufficient. Concerning the physicians' compliance with the guidelines, Rabe et al. ${ }^{12)}$ reported that the use of ICS in general hospitals as a preventative medication was low, even in patients with severe persistent asthma, ranging from 26\% in Western Europe to 9\% in Japan. The treatment in practice is rarely in con- formity with the guidelines in the management of asthma. Conformity with the guidelines in the university hospital as observed in the current study (78.0\%) was sufficiently higher than that in the previous reports, which allowed us to investigate the effect of guideline compliance on asthma control by excluding the lack of physicians' knowledge of the guidelines.

We selected ICS as a medication to observe patients' adherence as ICS is recommended as a firstline therapy and largely controls asthma. Several methods of measuring patients' ICS adherence have been reported, ${ }^{13)}$ including self-reported and questionnaire survey and electronic recording methods, but each method has its advantages and disadvantages. Self-report and questionnaire surveys have been used, giving a rather exact picture of patient medication adherence. In these methods, however, patients tended to report an overestimated adherence. Electronic measuring devices offer the most accurate and valid measurement of patient adherence. Adherence medication monitors, which can record the date and time of medication use, however, are expensive and must be adjusted to various types of equipment. Adherence can be distorted by episodes of a dead batteries or priming (i.e., a trial puff into the air before use). Furthermore, patients may be influenced by the presence of monitoring devices which can alter natural patterns of medication use, thus resulting in biased adherence.

We evaluated the patients' adherence by comparing the "expected" and "measured"' doses. ${ }^{9,10)}$ To calculate the "expected" doses, we used the physicians' prescribed orders as a daily dose as documented in the medical charts. The "measured" doses were calculated from the number of inhaled drugs dispensed in the pharmacy. In the case that the written integer number was not equal to the total number of prescribed orders, physicians ordered the integer number of inhaled drugs which is more than the total prescribed orders. If physicians continue to prescribe for a long time, upon requests by patients, they subsequently write the number of drugs which was reduced based on the amount of the drugs left, and the rest arose due to poor adherence. Consequently, for poorer patient medication adherence, the discrepancy is larger between the "expected" and "measured" doses. The method used in the present study is adequate for measuring patients' adherence with long-term medication regimens and may be a better method to meas- 
ure "true adherence" than other methods, which can be easily influenced by patients' efforts to deceive the investigators and/or patients' consciousness of being monitored, which often overestimate adherence. The average adherence in this study was $0.84 \pm 0.39$ (mean \pm S.D.), which was similar to the values $(0.74 \pm$ 0.32 ) reported in our previous study. ${ }^{8)}$

The number of outpatients selected as eligible during the surveillance was 50. Sample sizes were reviewed concerning the comparison between the patients with and without asthma medical history in their family, with a power of $80 \%$ and 2-sided $\alpha=$ 0.05 . Required sample sizes of 49 and 39 patients were calculated to detect a significant difference of the asthma-related emergency department visits' ratio and of patients' ICS adherence between the two groups, respectively. Therefore, these sample sizes were considered to be sufficient.

The introduction of JGL2003 may possibly affect the result of the comparison between at the beginning and at the end of asthma symptom severity, because JGL2003 was introduced during this surveillance. However, there was no significant difference in asthma symptom severity between at the beginning and at the end of surveillance. Consequently, there was little risk of bias due to the introduction of JGL2003 in the results of the present comparison between at the beginning and at the end of surveillance.

In JGL2006, some of the patients' factors are identified as the risk factors of asthma, i.e., asthma medical history in their family is one risk factor of asthma onset. Sunyer et al. ${ }^{14)}$ reported that parental asthma was associated with the patient's asthma, regardless of the age of asthma onset (odds ratio $=4.5$ ). Moreover, Siroux et al. ${ }^{15)}$ reported that active smoking is not a risk factor for asthma onset in adulthood, but smoking does increase asthma severity. In the present study, we identified an additional factor, a family history of asthma, that can significantly influence the patients' ICS adherence and the frequency of emergency department visits.

We compared the patients' ICS adherence between the presence and absence of family history of asthma, as shown in Fig. 1, and found a significant difference $(p=0.034)$. The frequency of unscheduled emergency visits tended to be low, and the number of emergency department visits was significantly low overall (Fig. 2). The patients with family medical histories of asthma were presumed to get easier access to correct medical information about the disease, which can be considered as one of the patient education. It is therefore necessary for medical providers to confirm patients' understanding of asthma, particularly for patients without a history of asthma in their family.

Concerning the patients with previous history of aspirin-induced asthma, atopic dermatitis, or allergic rhinitis, the frequency of unscheduled emergency visits tended to be higher (Fig. 2(a)), and the number of emergency department visits tended to be lower (Fig. 2(b)). The patients with previous history would be more anxious about their health than those without previous history. Therefore, the patients with previous history would tend to experience unscheduled emergency visit before the exacerbation. Concerning the patients with smoking habits, the frequency of emergency department visits tended to be higher than those without smoking habits (Fig. 2(b)), because of the fact that active smoking increases asthma severity (Table 2). ${ }^{15)}$

Several investigators have suggested that asthma symptom severity is associated with patients' adherence. ${ }^{16,17)}$ It was reported that patients were more adherent when the dose of ICS was increased and their asthma severity increased. ${ }^{18)}$ It was indicated that there was no overall significant relationship between the cumulative adherence and the final symptoms of surveillance. ${ }^{19)}$ There is a possibility that good asthma control ameliorates asthma symptom severity, which leads to improved patient adherence. Another possibility is that high patients' adherence leads to good asthma control and improves asthma symptoms. However, there has been no report that demonstrates the causality of these factors (i.e., patients' adherence and asthma symptom severity). As shown in Fig. 3, the mean patients' adherence to medication was not found to be correlated with asthma symptom severity at the beginning of the surveillance period, but was significantly correlated at the end of the surveillance. These results suggested that patients' adherence and asthma symptom severity are the cause and its consequence, respectively. Therefore, it is likely that high patient adherence leads to good asthma control and alleviates asthma symptom severity. This provides a reasonable basis to emphasize the importance of patients' good adherence for optimizing asthma therapy. Taylor et al. ${ }^{20)}$ reported that $31 \%$ of patients misunderstood that short-acting bronchodilator drugs were preventers, not relievers. 
Weinberger et al.21) also reported that patients receiving pharmaceutical care had significantly higher peak flow rates than the usual care group, and claimed that patient outcomes are enhanced by effective pharmacist-patient instructions.

Asthma symptom severity at the beginning of the surveillance in the physicians' noncompliant group was significantly higher than that in the compliant group. On the other hand, there was no significant difference between the two groups at the end of the surveillance period (Fig. 4). These results showed that the physicians' compliance dose not influence the asthma symptom severity, although the asthma symptom severity can influence the physicians' compliance with the guidelines. In other words, these results showed that asthma symptom severity was not exacerbated by physicians' noncompliance, and physicians' compliance to the guidelines was lower when asthma symptom severity was poorer. It does not appear that the treatment including divergence from the guidelines leads to an asthma control failure. Apparently, the guidelines did not appear to have any impact on the asthma severity. However, it might be interpreted by the following way. Physicians at the surveyed hospital do recognize the importance of the guidelines, and the divergence from the guidelines is thought to be an option for treatments when treatment failure is observed according to the guidelines. However, the results in Fig. 4 were obtained at only one university hospital, and one must be careful in applying the same results to other university hospitals, because the number of data used was relatively small.

Based on the above results, we proposed a flowchart for optimizing the asthma therapy (Fig. 5). Firstly, asthma control should be confirmed. If asthma symptom severity is low, changes in the treatment may not be necessary. Otherwise, the adherence of the patients should be checked, particularly after confirming whether they had asthma medical history in their family. For those who have no medical history of asthma in their family, an immediate asthma patient education course is necessary. Depending on the adherence, the patients may repeatedly participate in education program. Asthma control should be confirmed again in either case. If asthma control is poor, physicians' compliance to the guidelines must be confirmed, and the prescription should be reviewed. It is therefore necessary to once more confirm asthma control after a change in prescription.

In conclusion, we therefore advocate that patients' adherence to medication and physicians' compliance to the guidelines should be confirmed in order to have a better control over asthma, after carefully taking into account the patients' family medical history of asthma. Based on the present findings, a flow chart to optimize the asthma control was proposed. However, there is a limitation of our recommendations as implemented in the flow chart, because the results were obtained from a surveillance of a patient series in only

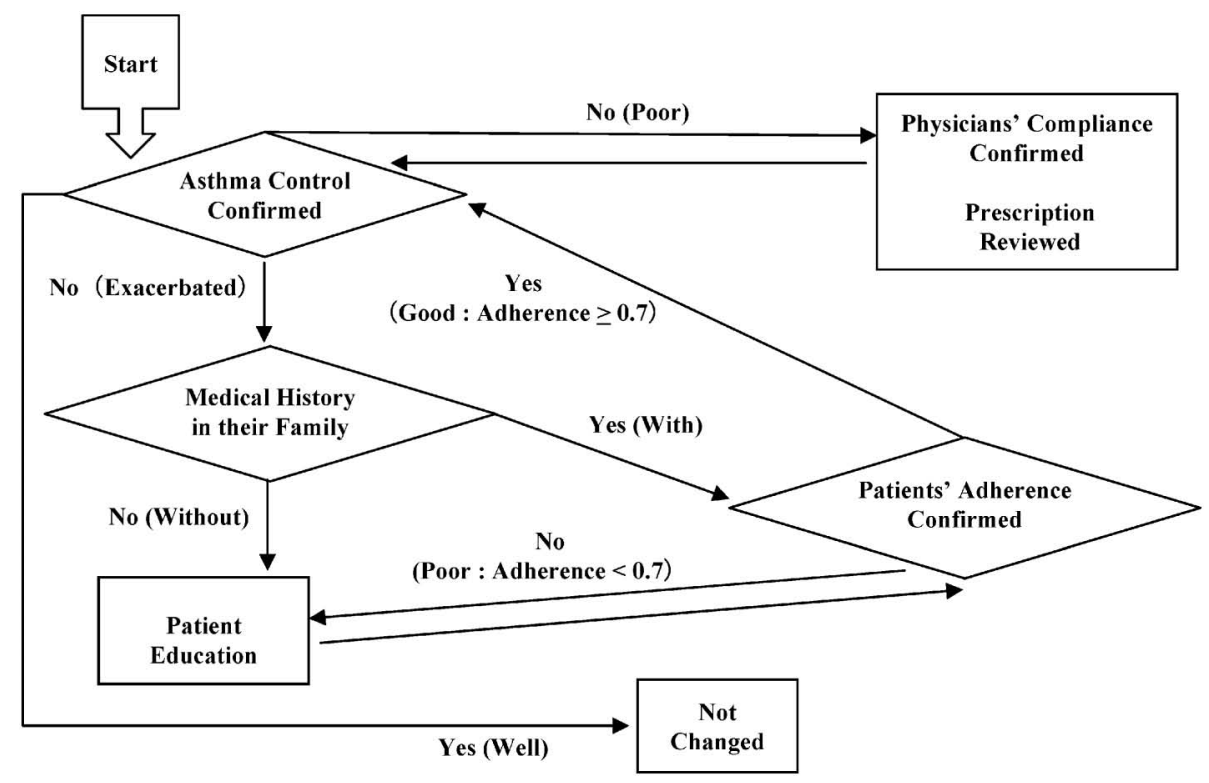

Fig. 5. A Proposed Flow Chart for Asthma Treatment 
one university hospital, where physicians' compliance to guidelines are expected to be better than that found in a general hospital.

Acknowledgements This study was supported by a High-Technology Research Grant provided by Showa University.

\section{REFERENCES}

1) WHO/NHLBI Workshop Report, Global Strategy for Asthma Management and Prevention, 1995. Update 2008: Global Initiative for Asthma (GINA) : http://www.ginasthma.org, cited 17 June, 2009.

2) Japanese Society of Allergology, Asthma Prevention and Management Guidelines 2006, Kyowa Kikaku, Tokyo, 2006.

3) Suissa S., Ernst P., Benayoun S., Baltzan M., Cai B., N. Engl. J. Med., 343, 332-336 (2000).

4) de Marco R., Cazzoletti L., Cerveri I., Corsico A., Bugiani M., Accordini S., Carrozzi L., Dallari R., De Togni A., Marinoni A., Pirina P., Janson C.; ISAYA Study Group, Int. Arch. Allergy Immunol., 138, 225-234 (2005).

5) Williams L. K., Pladevall M., Xi H, Peterson E. L., Joseph C., Lafata J. E., Ownby D. R., Johnson C. C., J. Allergy Clin. Immunol., 114, 1288-1293 (2004).

6) Suzuki T., Hasegawa T., Suzuki E., Sasahara K., Kawada T., Koya T., Akazawa K., Satoh H., Gejyo F., Allergy Asthma Proc., 24, 347351 (2003).

7) Barr R. G., Somers S. C., Speizer F. E., Camargo C. A. Jr., Arch. Intern. Med., 162, 1761-1768 (2002).

8) Suzuki T., Hasegawa T., Kawada T., Suzuki E., Satoh H., Gejyo F., Sato S., Nishigaki R., Jpn. J. Pharm. Health Care Sci., 33, 221-228 (2007).

9) Kelloway J. S., Wyatt R. A., Adlis S. A., Arch. Intern. Med., 154, 1349-1352 (1994).
10) Gallefoss F., Bakkep P. S., Am. J. Respir. Crit. Care Med., 160, 2000-2005 (1999).

11) Lagerlùv P., Veninga C. C. M., Muskova M., Hummers-Pradier E., StaÊ 1sby Lundborg C., Andrew M., Haaijer-Ruskamp F. M., Eur. Respir. J., 15, 25-29 (2000).

12) Rabe K. F., Adachi M., Lai C. K. W., Soriano J. B., Vermeire P. A., Weiss K. B., Weiss S. T., J. Allergy Clin. Immunol., 114, 40-47 (2004).

13) Rand C. S., Wise R. A., Am. J. Respir. Crit. Care Med., 149, S69-76 (1994).

14) Sunyer J., Antó J. M., Kogevinas M., Barceló M. A., Soriano J. B., Tobías A., Muniozguren N., Martínez-Moratalla J., Payo F., Maldonado J. A., Eur. Respir. J., 10, 2490-2494 (1997).

15) Siroux V., Pin I., Oryszczyn M. P., Moual N. Le., Kauffmann F., Eur. Respir. J., 15, 470477 (2000).

16) Barr R. G., Somers S. C., Speizer F. E., Camargo C. A. Jr., Arch. Intern. Med., 162, 1761-1768 (2002).

17) Diette G. B., Wu A. W., Skinner E. A., Markson L., Clark R. D., McDonald R. C., Healy J. P., Huber Jr. M., Steinwachs D. M., Arch. Intern. Med., 159, 2697-2704 (1999).

18) Wraight J. M., Cowan J. O., Flannery E. M., Town G. I., Taylor D. R., Respirology, 7, 133 -139 (2002).

19) Kim C., Feldman H. I., Joffe M., Tenhave T., Boston R., Apter A. J., J. Allergy Clin. Immunol., 115, 810-814 (2005).

20) Taylor D. M., Auble T. E., Calhoun W. J., Mosesso Jr, V. N., Chest, 116, 1638-1645 (1999).

21) Weinberger M., Murray M. D., Marrero D. G., Brewer N., Lykens M., Harris L. E., Seshadri R., Caffrey H., Roesner J. F., Smith F., Newell A. J., Collins J. C., McDonald C. J., Tierney W. M., JAMA, 288, 1594-1602 (2002). 